\title{
Isolation and Inoculation of Endophytic Actinomycetes in Root Nodules of Elaeagnus Angustifolia
}

\author{
Mingsheng Chen \\ College of Life Science, Hebei University \\ Key Lab of Microbial Diversity Research and Application of Hebei Prov., Baoding 071002, China \\ E-mail: cms-8528@163.com
}

Liping Zhang (Corresponding author)

College of Life Science, Hebei University

Key Lab of Microbial Diversity Research and Application of Hebei Prov., Baoding 071002, China

Tel: 86-0312-5079696 E-mail: zhlping@hbu.edu.cn

\author{
Xiumin Zhang \\ College of Life Science, Hebei University \\ Key Lab of Microbial Diversity Research and Application of Hebei Prov., Baoding 071002, China
}

Received: December 28, 2010

Accepted: March 7, 2011

doi:10.5539/mas.v5n2p264

This research was financially supported by National Science Foundation of China titled "studies on taxonomy of symbiotic fixation actinomycetes (Grant No.30770003) "and Technological conditions construction program of Hebei Province (Grant No.10967146D).

\begin{abstract}
8 strains of endophytic actinomycetes were successfully isolated from root nodules of Elaeagnus angustifolia by the methord of nodule-slicing respectively on three mediums S, FTW and DPM. And these strains were analyzed by $16 \mathrm{~S}$ rDNA phylogenetic studies, the result showed that 5 of these strains belonged to Micromonospora, and the other 3 strains was Nonomuraea, Pseudonocardia and Planotetraspor, repectively.Inoculation of these isolated strains to the host plant, although no root nodules were obtained, the growth degree of the plant was obviously increased compared with the control.
\end{abstract}

Keywords: Elaeagnus angustifolia, Endophytic actinomycetes, Phylogenetic analysis, Inoculation

Elaeagnus angustifolia L., also known as cinnamon Liu, Yin Liu, belongs to Elaeagnaceae Elaeagnus (Qian, 1983, PP. 40-41). It mainly distributed in northwest of China and western inner Mongolia, and possessed the characteristics of strong vitality, drought-resistance, wind-resistance, salinity resistance, tolerant of poor soils and so on. Its lateral root developed very well with large root pieces, could grow a lot of nodules which played roles in improving soil fertility and ameliorating soil. Ever since a pure culture (CPI-l) of endophyte was first isolated from Comptonia peregrina (Callaham, 1978, PP. 899-902), a large number of pure cultures have been isolated from different nodule plants with different actinomycetes all around the world, but such studies on Elaeagnus angustifolia. were less documented. In the present paper, endophytic actinomycetes from Elaeagnus angustifolia were isolated and incubated, and inoculation of nodules was investigated. We attempted to reveal species diversity of endophytic actinomycetes from Elaeagnus angustifolia, and specie resource of abundant actinomycetes, further understand biological characteristics of these endophytic actinomycetes and effects of them on plants, and provide valuable reference information.

\section{Materials and methods}

\subsection{Nodules source}

Elaeagnus root nodules were all collected from Chifeng Botanic Gardens of Inner Mongolia Autonomous Region, rinsed by clean water, packed according to nodule clusters. After numbered, they were preserved at 
$-20{ }^{\circ} \mathrm{C}$. The serial number used in the present paper was ZW-1, ZW-2 and ZW-3.

\subsection{Isolation of pure culture}

\subsubsection{Isolation medium}

S (Lechevalier, 1983, PP. 2826-2831), FTW (Simonet, 1985, PP. 49-60) and DPM (Baker, 1984, PP. 23-28) mediums were applied in the present paper.

\subsubsection{Separation}

Proper amount of nodules were rinsed by tap water, its impurities and old nodules were removed. Its flaps were cut down, washed by sterile water three times, placed into $75 \%(\mathrm{v} / \mathrm{v})$ of ethanol for 5-6 min by forceps, transferred into $0.1 \%$ mercuric chloride to disinfect for $2 \mathrm{~min}$, and rinsed by sterile water three times. When the surface was disinfected, nodules were placed into aseptic mortar, and ground into homogenate-like solution. A certain amount of homogenate was coated on the surface of three mediums, viz. S, FTW and DPM, and then incubated at $28{ }^{\circ} \mathrm{C}$. Colony growth was observed once two days, and its initial time and characteristics were recorded.

\subsection{Strain preservation and cell culture}

The isolated pure culture was inoculated into $\mathrm{S}$ liquid medium, and shaking incubated at $28{ }^{\circ} \mathrm{C}$ for 5-7 days. When strains grew to the logarithmic phase, and tested by microscope to check its purity. Proper amount of pure culture was added to glycerintubes containing 30 to $50 \%$ glycerol, and preserved at $-70{ }^{\circ} \mathrm{C}$. The other culture was centrifuged to collect cells, and the cells were preserved at $-20{ }^{\circ} \mathrm{C}$ for further investigation.

\subsection{Extraction of genomic DNA}

Genomic DNA was extracted by the modification of Fred method (Baker, 1986, PP. 233-236).

\subsection{PCR amplification of $16 \mathrm{~S}$ rDNA sequence}

\subsubsection{PCR amplification of 16S rDNA sequence}

Using 16S rDNA sequences universal primers 27f (E.coli 8-27 bases: 5'-GAG TTT GAT CCT GGC TCA G-3') and 1525r (E.coli 1525-1545 bp: 5'-AGA AAG GAG GTG TAC CAGCC-3') as primers, 16S rDNA was undertaken PCR amplification, and PCR products were sequenced by Beijing Sinogene Biotech Co.Ltd.

\subsection{Construction of phylogenetic tree}

The obtained 16S rDNA sequences was undertaken homology comparison with the known sequences of Genbank database using BLAST method, and multiple sequence alignment by Clustal W1.4 (Thompson, 1994, PP. 4673-4680). Phylogenetic trees were constructed using neighbor joining module of software MEGA4.1 (Tamura, 2007, PP. 1596-1599) in order to analysis phylogeny of strains.

\subsection{Inoculation of pure culture}

\subsubsection{Angustifolia seed germination}

Angustifolia seeds were washed with tap water to remove the surface impurities, and then soaked into the warm water of $40{ }^{\circ} \mathrm{C}$ for $24 \mathrm{~h}$. During the immersion process, tap water was replaced once. The surface should be sterilized before seeds germinated, the seeds were added to culture dishes with sterile filter paper, and proper amount of water was added. Seeds were cultured at $30{ }^{\circ} \mathrm{C}$ till germination. When the shoots grew up to $1-2 \mathrm{~cm}$, inoculation was taken immediately.

\subsubsection{Inoculation of pure culture}

The pure cultures of the isolated 8 strains endophytes were inoculated into $\mathrm{S}$ liquid medium, cultured for $7 \mathrm{~d}$, rinsed by sterilized water, centrifuged to collect cells and made into bacterial suspension after homogenization.

(2) Seedlings of sterile cultured Elaeagnus was immersed into bacterial suspension for $2 \mathrm{~h}$, and then transferred into large test tubes with nitrogen-free Hoagland nutrient solution. They were incubated after well marked, and treatment without inoculation was applied as blank control. Plant growth was regularly observed.

\section{Results and analysis}

\subsection{Isolation results of Elaeagnus nodules}

8 strains of actinomycetes were successfully isolated from 3 Elaeagnus nodules samples. Among them, strains D30401, D30202, C20201 and C10404 were obtained from S, D10204, C10401 and D30407 from FTW, and D30511 from DPM. 


\subsection{Phylogenetic trees construction of the isolated straom 16S rDNA}

By comparing the isolated strains and standard strain $16 \mathrm{~S}$ rDNA sequences of relative genus in GenBank database, 8 strains belonged to 4 genus, including 5 Micromonospora, 1 Pseudonocardia, 1 Planotetraspora, and 1 Nonomuraea. Phylogenetic trees of the constructed 16SrDNA sequences was depicted in Figure 1. Phylogenetic results indicated that the greatest similarity of strains D30401, D30202 and D30511with 16S rDNA sequences of Micromonospora endolithica was $99.5 \%, 99.6 \%$ and $99.6 \%$, respectively; the greatest similarity of strains C10401 and D30407 with 16S rDNA sequences of Micromonospora coxensis was both $99.6 \%$; the greatest similarity of strains C20201 with 16S rDNA sequences of Pseudonocardia carboxydivorans was 100\%; the greatest similarity of strains C10404 with 16S rDNA sequences of Planotetraspora mira was 99.8\%; the greatest similarity of strains D10204 with 16S rDNA sequences of Nonomuraea helvata was $99.1 \%$.

\subsection{Inoculation results}

Inoculation results indicated that 8 strains failed to nodule after they were inoculated back to host plants for $70 \mathrm{~d}$, but plant mass growth was significantly higher than the control, which all showed a manner as follows: the whole plant grew vigorously, and the height of stem was significantly higher than the control, and root system was stronger than the control. Inoculation result of D30202 was depicted in Figure 2.

\section{Discussions}

In the present study, 8 strains of actinomycetes were successfully isolated from Elaeagnus angustifolia in the nodule structure by three mediums using nodule-slicing method. As seen from phylogenetic analysis of $16 \mathrm{~S}$ rDNA sequence results, 8 strains belonged to 4 genus, which indicated that endophytic actinomycetes from Elaeagnus angustifolia had abundant diversity, and Micromonospora dominated. After the isolated actinomycetes from nodules were inoculated back into host plants, they failed to induce nodules of host plants, which suggested that the suitable condition of pure culture back inoculation still need further investigation. The mass growth increased significantly compared with the control, which indicated that the presence of these endophytes had certain promoting effects on plants. However, its biological characteristics, how to induce nodules, and whether to possess nitrogen fixation activities still need further investigation.

\section{References}

Baker, D., \& Danell, K.H. (1986). Effects of oxygen and chloramphenicol on Frankia nitrogenase activity. Arch Microbiol, 144:233-236.

Baker, D., \& O'Keefe, D. (1984). A modified sucrose fractionation procedure for the isolation of frankiae from actinorhizal root nodules and soil samples. Plant and Soil, 78:23-28.

Callaham, D., Tredici, P.D., \& Torrey, J.G. (1978). Isolation and cultivation in vitro of the actinomycete causing root nodulation in Comptonia. Science, 199:899-902.

Lechevalier, M.P., Baker, D., \& Horriere. (1983). Physiology,chemistry,serology,and infectivity of two Frankia isolates from Alnusincana sub sp. Rugosa Can J Bot, 61:2826-2831.

Qian, C.S., Chen, H.Y., \& Lin, R., et al. (1983). Chinese flora. Beijing: Science Press, 40-41.

Simonet, P., Normand, P., \& Moiroud, A., et al. ( 985$)$. Restriction enzyme digestion of Frankia plasmids. Plant and Soil, 87:49-60.

Tamura, K., Dudley, J., \& Nei, M. (2007). MEGA4:Molecular Evolutionary Genetics Analysis(MEGA)software version 4.0. Molecular Biology and Evolution, 24:1596-1599.

Thompson, J.D., Higgins, D.G., \& Gibson, T.J. (1994). CLUSTAL W: improving the sensitivity of progressive multiple sequence alignment through sequence weighting,position-specific gap penalties and weight matrix choice. Nucleic Acids Res Nov., 11, 22(22):4673-4680. 


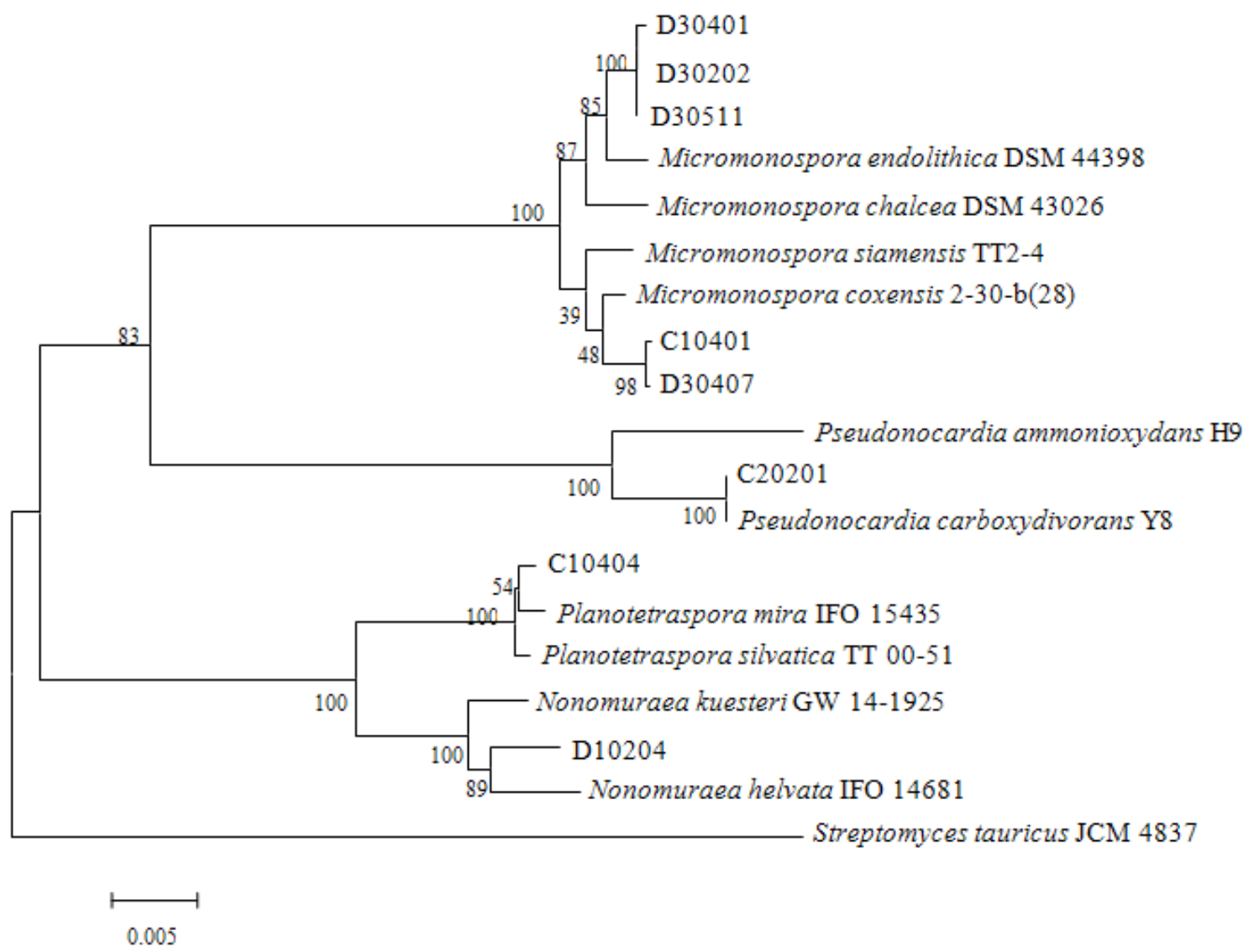

Figure 1. Neighbour-joing tree based on 16S rDNA sequences between the strains tested and related strains

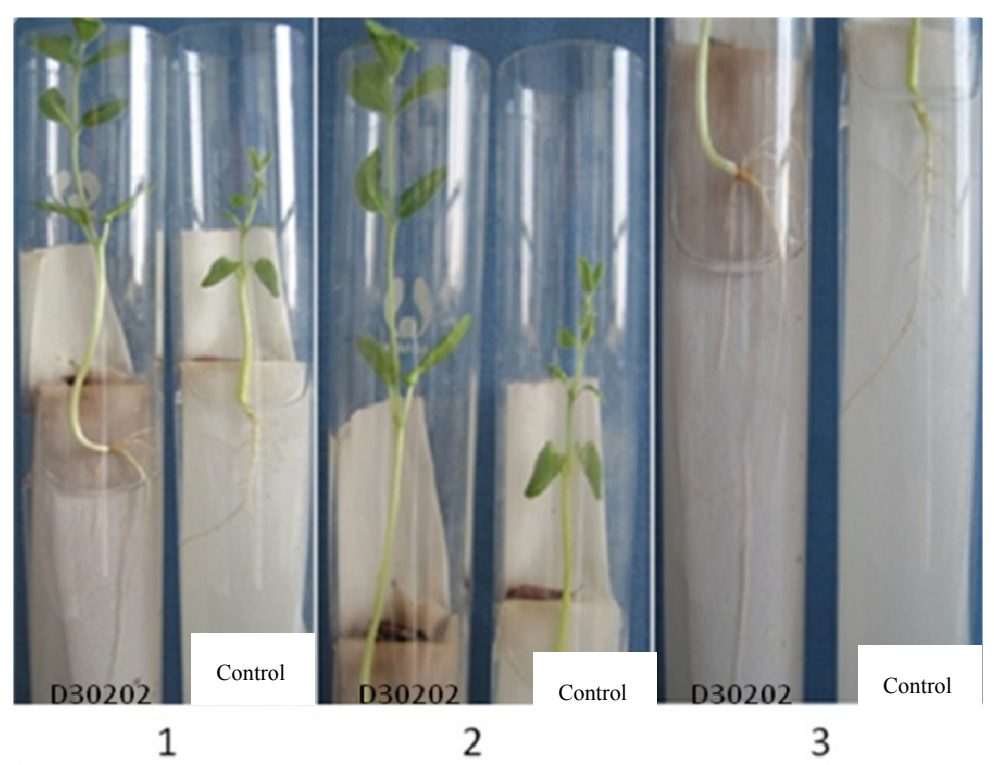

Figure 2. Inoculation result of stain D30202

Note: 1 , the whole plant; 2 , stem; 3 , root. 